\title{
Virtual Behavioural Medicine Program: A Novel Model of Care for Neuropsychiatric Symptoms in Dementia ${ }^{1}$
}

\author{
Morris Freedman ${ }^{\mathrm{a}, \mathrm{b}, *}$, Malcolm A. Binns ${ }^{\mathrm{b}, \mathrm{c}}$, Fidelma Serediuk ${ }^{\mathrm{d}}$, M. Uri Wolf ${ }^{\mathrm{e}}$, Einat Danieli ${ }^{\mathrm{d}}$, \\ Bradley Pugh ${ }^{\mathrm{b}, \mathrm{d}}$, Deb Galet ${ }^{\mathrm{d}}$, Eslam Abdellah ${ }^{\mathrm{d}, 2}$, Ericka Teleg ${ }^{\mathrm{d}}$, Mindy Halper ${ }^{\mathrm{d}}$, Lauren Masci ${ }^{\mathrm{d}}$, \\ Adrienne Lee ${ }^{\mathrm{d}}$ and Anne Kirstein ${ }^{\mathrm{d}}$ \\ ${ }^{a}$ Department of Medicine (Neurology), Baycrest Health Sciences, Mt. Sinai Hospital, and University of Toronto, \\ Ontario, Canada \\ ${ }^{\mathrm{b}}$ Rotman Research Institute of Baycrest Centre, Toronto, Ontario, Canada \\ ${ }^{\mathrm{c}}$ Dalla Lana School of Public Health, University of Toronto, Ontario, Canada \\ ${ }^{\mathrm{d}}$ Baycrest Health Sciences, Toronto, Ontario, Canada \\ ${ }^{\mathrm{e}}$ Department of Psychiatry, Baycrest Health Sciences and University of Toronto, Ontario Canada
}

Handling Associate Editor: Amos Korczyn

Accepted 12 January 2022

Pre-press 14 February 2022

\begin{abstract}
.
Background: Patients with severe neuropsychiatric symptoms (NPS) due to dementia are often uprooted from their familiar environments in long-term care or the community and transferred to emergency departments, acute care hospitals, or specialized behavioral units which can exacerbate NPS. To address this issue, we developed the Virtual Behavioural Medicine Program (VBM), an innovative model of virtual care designed to support management of patients with NPS in their own environment.

Objective: To determine efficacy of VBM in reducing admission to a specialized inpatient neurobehavioral unit for management of NPS.

Methods: We reviewed outcomes in the first consecutive 95 patients referred to VBM. Referrals were classified into two groups. In one group, patients were referred to VBM with a simultaneous application to an inpatient Behavioural Neurology Unit (BNU). The other group was referred only to VBM. The primary outcome was reduction in proportion of patients requiring admission to the BNU regardless of whether they were referred to the BNU or to VBM alone.

Results: For patients referred to VBM plus the BNU, the proportion needing admission to the BNU was reduced by $60.42 \%$. For patients referred to VBM alone, it was $68.75 \%$.

Conclusion: VBM is a novel virtual neurobehavioral unit for treatment of NPS. Although the sample size was relatively small, especially for the VBM group, the data suggest that this program is a game changer that can reduce preventable emergency department visits and acute care hospital admissions. VBM is a scalable model of virtual care that can be adopted worldwide.
\end{abstract}

Keywords: Behavioral and psychological symptoms of dementia, dementia, models of care, neuropsychiatric symptoms, responsive behaviors, telehealth, virtual care

\footnotetext{
${ }^{1}$ The work reported in this paper was presented in part at the International Psychogeriatric Association Virtual Congress, 2021.

${ }^{2}$ Current affiliation: Hamilton General Hospital, Hamilton, Ontario, Canada.

*Correspondence to: Morris Freedman, MD, FRCPC, Baycrest Health Sciences, 3560 Bathurst Street, Kimel Family Building,
}

Rm 656, Toronto, Ontario M6A 2E1, Canada. Tel.: 4167852500 ext. 2400; E-mail: mfreedman@baycrest.org. 


\section{INTRODUCTION}

The number of people diagnosed with dementia worldwide is expected to triple from 50 to 150 million by 2050 [1]. Severe neuropsychiatric symptoms (NPS) in advanced dementia, such as aggression and agitation [2], contribute to this growing crisis by adversely impacting individuals with dementia, their families, and healthcare systems.

NPS, also referred to as behavioral and psychological symptoms of dementia (BPSD) [3] and responsive behaviors [4], are often the driver for patients being uprooted from their familiar environment in long-term care (LTC) or the community and transferred to specialized transitional behavioral units [5], emergency departments, or acute care hospitals. Relocating patients to unfamiliar surroundings often escalates NPS due to changes in environment [6]. Moreover, emergency department transfers often result in acute care hospital admissions which can further contribute to cognitive and functional decline [7] and exacerbate NPS.

To reduce the need for transfers to specialized transitional behavioral units, emergency departments, or acute care hospitals, we developed an innovative model of care designed to assess and manage patients with NPS virtually while they remain in their own familiar environment. We also sought to support acute care hospitals in the management of patients with NPS. These behaviors pose barriers for discharge to LTC, resulting in increased length of stay, as well as triggering transfers to specialized neurobehavioral units.

The new model of care, called the Virtual Behavioural Medicine Program (VBM), is in essence a "virtual hospital inpatient neurobehavioral unit" for assessment and management of NPS. Since VBM is virtual, it is not limited by physical space or number of available beds. Although VBM was developed pre-COVID-19, its growth was facilitated by the pandemic due to the unique virtual care benefits that it offered to patients, caregivers, and healthcare staff in LTC, acute care hospitals, and the community.

Although the concept of virtual hospital care is not new, the focus of VBM on NPS due to dementia represents a novel application of the virtual hospital concept. In this regard, Lee et al. [8] also used telehealth to care for patients with dementia who resided in facilities or the community. However, their primary purpose was to provide care for patients with dementia in settings where specialists were not available, as opposed to focusing specifically on NPS. Virtual hospital care has recently also been used for patients with COVID-19 [9-11].

A comment is warranted about hospitals-at-home [7, 12-20]. This concept involves provision of healthcare services to patients in the community as a substitute for inpatient hospital admission. It also involves home visits by the healthcare team from the host site. Whereas the VBM model is similar to the hospital-at-home concept with respect to care of patients in the community, VBM differs in that it also focuses on patients in LTC and acute care hospitals. Moreover, VBM is entirely virtual.

Hospitals at home have focused on disorders that include congestive heart failure, chronic obstructive airway disease, community acquired pneumonia, fluid and electrolyte disorders, urinary tract infections, acute ischemic stroke, cellulitis, deep vein thrombosis, pulmonary embolism, asthma, complicated urinary tract infection or urosepsis, nausea, vomiting, and dehydration $[7,12,13,15,17,18$, $20,21]$.

Our research goal was to determine the efficacy of VBM in reducing admissions of patients referred to specialized inpatient behavioral units for management of NPS. Prior to development of VBM, patients referred to the inpatient Behavioural Neurology Unit at Baycrest (BNU) typically remained on the waitlist without significant improvement until they were admitted and treated as inpatients. Moreover, some cases were on the waitlist for a year or more. Our hypothesis was that VBM would be highly effective in reducing the need for inpatient admission. This was tested by retrospectively reviewing the outcomes of the first consecutive ninety-five patients referred to the program. In addition to reporting our research findings, we describe the VBM program and how it was developed.

\section{METHODS}

The study was approved by the Research Ethics Board at Baycrest Health Sciences.

\section{VBM program development and description}

VBM was developed as an extension of the ambulatory Sam and Ida Ross Memory Clinic at Baycrest Health Sciences. The initial goal was to reduce the waitlist for patients requiring admission to the inpatient BNU for management of severe NPS by treating these behaviors virtually. This was later extended to 
assessment and management of patients with NPS who were referred to VBM without a parallel referral to the inpatient BNU. Nevertheless, some of these patients had NPS that were severe enough to warrant an inpatient application.

VBM is a collaborative partnership between the Baycrest Sam and Ida Ross Memory Clinic and the Toronto Central Behavioural Support for Seniors Program (TC BSSP). The TC BSSP is a locally lead program in the Toronto Central Region under the overall umbrella of the provincial Behavioural Supports Ontario (BSO) program. BSO is mandated to build capacity for management of NPS through evidenced based outreach services including assessment, care planning and coaching through implementation, system integration and coordination, education, and training. The core BSO clinical work focuses on non-pharmacological approaches including behavioral care plans, communication strategies, and environmental modification. The BSO teams also work with Geriatric Psychiatry Outreach Teams in the community and LTC. The TC BSSP Coordination Office works in collaboration with VBM by linking to the Behaviour Supports Team where the patient is located. Patients referred to VBM require pharmacological treatment in addition to non-pharmacological management.

The VBM core team is comprised of physicians (behavioral neurologist, geriatric psychiatrist, and clinical behavioral neurology fellows), a registered nurse, pharmacist, BSO clinical navigator, and BSO allied health practitioners. The VBM team works in close collaboration with healthcare teams in LTC, acute care, and the community. These teams include primary care physicians, psychiatrists, geriatricians, internists, physiatrists, behavioral support staff, nurses, occupational therapists, care coordinators, social workers, recreational therapists, and family members.

The technologies used in VBM were primarily videoconferencing, although telephone was used when there were technical difficulties with videoconferencing. In addition, some participants joined the videoconference by telephone.

Patients were referred to VBM from LTC, acute care, or the community for virtual assessment and management of NPS such as physical aggression. Patients in the community were in a private residence or a congregate setting outside of LTC such as a retirement home or prison. Referrals to VBM were classified into two groups. In one group, patients were referred only to VBM for virtual assessment and management of NPS. The other group was referred to VBM with a simultaneous application to the inpatient BNU at Baycrest. These patients were classified as BNU applicants. Thus the classification of patients as being referred to BNU or only VBM depended on the request of the referral source. For the BNU group, the goal was to attempt treatment virtually and admit patients to the inpatient unit only if virtual management was not successful. The BNU is a 20-bed specialized neurobehavioral unit for patients with NPS and has been described elsewhere [5].

An important characteristic of the VBM team is that the behavioral neurologist and geriatric psychiatrist in VBM also work on the BNU. Thus, the VBM model facilitated continuity of medical care for those patients who required admission to the BNU. It also ensured that the medical expertise, as well as the pharmacological strategies employed in VBM, were the same in VBM as on the BNU.

\section{Initial assessment, virtual patient rounds, and virtual waiting room}

Patients referred to VBM were initially seen in consultation virtually by the physician. Wait times ranged from approximately 1-3 weeks. For follow-up, and in keeping with standard practice on physical inpatient units, we implemented regular patient rounds. During these rounds, the VBM team saw the patients and met with the healthcare teams, family, and caregivers, all through virtual means. The purpose of the rounds was to review patient progress, monitor therapy, make changes in medication as indicated, and review non-pharmacological strategies that may have been implemented by the BSO team or other healthcare staff. Depending on individual patient needs, the virtual rounds occurred as often as weekly. In addition, the VBM physicians and nurse were available between scheduled visits by telephone or email.

We implemented a virtual waiting room where VBM administrative staff monitored the patient schedule and informed healthcare teams in LTC and acute care, as well family and caregivers in the community, if the VBM team was running late. In these cases, the administrative staff informed those waiting that they would be contacted by phone when the VBM team were ready for the virtual visit. This allowed staff at the remote sites, as well as family and caregivers, to attend to other duties while waiting for the VBM rounds visit. 
Table 1

Severity Rating Scale

\begin{tabular}{ll}
\hline Rating & Definition \\
\hline 1 & Needs admission with high physical aggression. \\
2 & Needs admission with moderate physical aggression. \\
3 & Needs admission for non-physical NPS. \\
4 & Need for admission unclear since NPS may be \\
& manageable without admission. \\
5 & Probably does not need admission. \\
6 & No significant NPS and thus does not need admission \\
& but remains on wait list. \\
& Does not need admission and can be removed from wait \\
& list. \\
\hline
\end{tabular}

\section{VBM study review process}

A retrospective review of all consecutive patients assessed in VBM from inception of the program on February 14, 2020 until December 24, 2020 was undertaken. All patients had been rated according to a 7-point Severity Rating Scale that was developed (Table 1). This scale was administered after the initial assessment and updated during follow-up. Ratings 1, 2 , or 3 indicate a clear need for admission to the BNU. Ratings 4 or 5 indicate that admission is possibly or probably not required. Ratings 6 or 7 indicate that admission to the BNU is not required. The difference between ratings 6 and 7 is that a rating of 6 indicates that the patient is ready for discharge, but that discharge had not yet taken place. A rating of 7 indicates discharge from VBM. If an application to the inpatient BNU was made, a rating of 7 also indicates that the patient has been removed from the inpatient waiting list. The primary outcome measure was the reduction in need of admission to the inpatient BNU regardless of whether they were referred to the BNU or to VBM alone. As stated previously, the patients in the BNU group were referred to BNU plus VBM.

Follow-up visits were defined as scheduled virtual visits by videoconference or phone, as well as unscheduled but urgent virtual visits by videoconference or phone. Brief phone updates with healthcare professionals in acute care, LTC, or the community were not counted as follow-up visits.

\section{Analysis}

We used analysis of variance to compare age related to referral source and referral target. Logistic regression was used to determine whether sex distribution was different between referral source and referral target. Ordinal logistic regression was used to model the Severity Rating Scale measure.
Bootstrap resampling was used to estimate a 95\% confidence interval around the estimate for the within-subject proportional reduction in the need for hospitalization.

In order to examine duration in the program, we fit Cox proportional hazards models using two measures of time: number of days in the program and number of follow-up visits. The target event was defined as discharge from VBM, i.e., a final rating of 7. For those with a BNU application, this also included discharge from the BNU waitlist. If a participant was discharged prior to the target event, then they were identified as censored, and we recorded the time of observation until that point.

Analyses were run in $\mathrm{R}$ version 3.6.1. Analysis of variance and logistic regression were run using the glm package. Ordinal logistic regression was run using the polr package. Cox proportional hazards model was run using the coxph package. All hypothesis tests were performed at an alpha level of $5 \%$. The Benjamini-Hochberg procedure was used to control the false discovery rate for all comparisons between the three referral sources. We report adjusted $p$-values for the referral source factor with subscript indicating order of magnitude of the original $p$-value: $p_{i}=\frac{3}{i} \cdot p_{(i)}$ where $\mathrm{p}_{(1)}$ is the smallest $p$-value.

\section{RESULTS}

In support of our hypothesis, among the patients referred to the inpatient BNU the proportion who required admission was reduced by $60.42 \%$. Prior to presenting the analyses related to this finding, we shall describe the characteristics of the patient sample and duration in VBM. Figure 1 shows a flow chart of the number of patients seen at different stages.

\section{Participants referred to the VBM program}

Table 2A shows characteristics of the patient sample referred to VBM during the study period. Ninety-five cases from LTC, acute care, or the community were referred to VBM or to both VBM and the inpatient BNU. VBM will be used to describe the former target program and BNU will be used for the latter target program.

Patients referred from acute care were significantly younger than those from LTC $\left(\mathrm{p}_{1}=0.0002\right)$ and the community $\left(\mathrm{p}_{2}=0.0089\right)$. There were two patients from acute care in their $40 \mathrm{~s}$, whereas the two youngest patients in LTC and the community were 56 and 61 


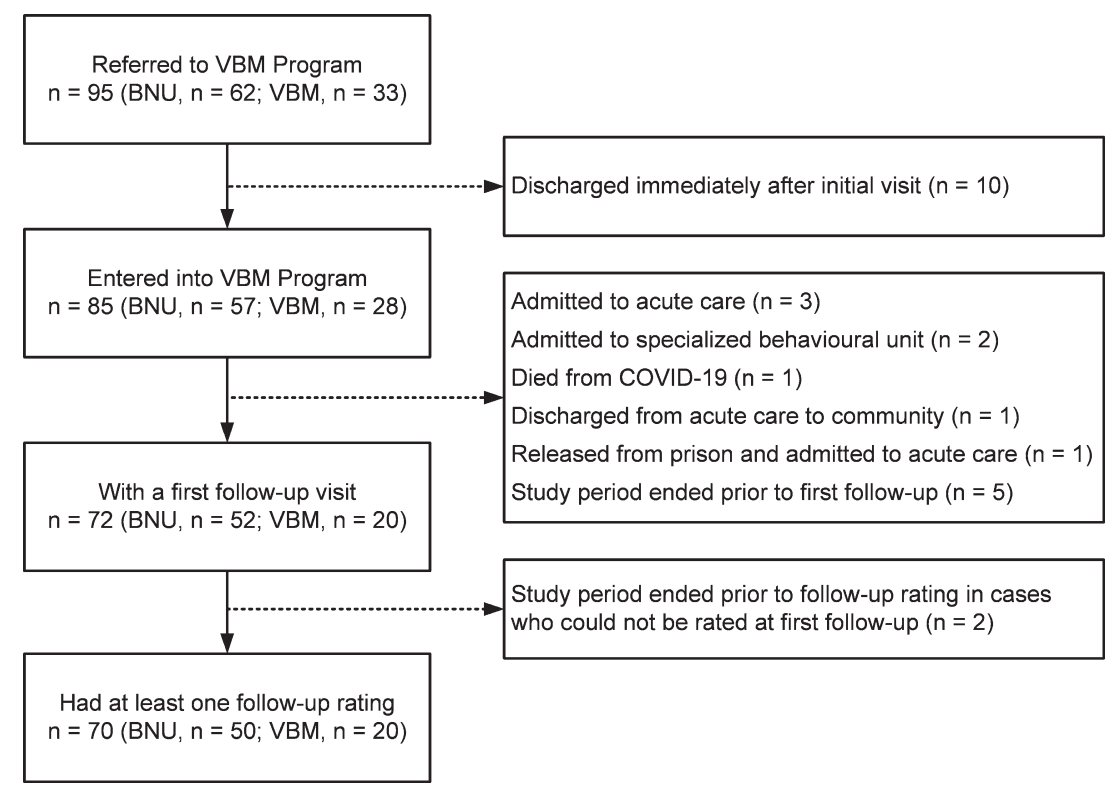

Fig. 1. Flow chart of the number of patients seen at different stages.

Table 2A

Patient Demographics (Full Sample)

\begin{tabular}{|c|c|c|c|c|c|c|}
\hline & \multirow[b]{2}{*}{$\begin{array}{c}\text { Total Sample } \\
n=95\end{array}$} & \multicolumn{3}{|c|}{ Referral Source } & \multicolumn{2}{|c|}{ Target Program } \\
\hline & & $\begin{array}{c}\text { Acute Care } \\
n=35\end{array}$ & $\begin{array}{c}\text { LTC } \\
n=30\end{array}$ & $\begin{array}{c}\text { Community } \\
n=30\end{array}$ & $\begin{array}{l}\mathrm{BNU} \\
n=62\end{array}$ & $\begin{array}{l}\text { VBM } \\
n=33\end{array}$ \\
\hline Age, mean (SD) & $73.5(11.7)$ & $67.5(13.4)$ & $78.6(9.1)$ & $75.5(8.7)$ & $73.2(11.7)$ & $74.2(11.9)$ \\
\hline Sex, M:F & $57: 38$ & $28: 7$ & $15: 15$ & $14: 16$ & $40: 22$ & $17: 16$ \\
\hline Patients with follow-ups, $n(\%)$ & $72(75.8 \%)$ & $26(74.3 \%)$ & $26(86.7 \%)$ & $20(66.7 \%)$ & $52(83.9 \%)$ & $20(60.6 \%)$ \\
\hline
\end{tabular}

BNU, Behavioural Neurology Unit; VBM, Virtual Behavioural Medicine Program.

Table 2B

Demographics of Patients Entered into Program

\begin{tabular}{|c|c|c|c|c|c|c|}
\hline & \multirow[b]{2}{*}{$\begin{array}{c}\text { Total Sample } \\
\quad n=85\end{array}$} & \multicolumn{3}{|c|}{ Referral Source } & \multicolumn{2}{|c|}{ Target Program } \\
\hline & & $\begin{array}{c}\text { Acute Care } \\
n=32\end{array}$ & $\begin{array}{c}\text { LTC } \\
n=28\end{array}$ & $\begin{array}{c}\text { Community } \\
n=25\end{array}$ & $\begin{array}{l}\mathrm{BNU} \\
n=57\end{array}$ & $\begin{array}{l}\text { VBM } \\
n=28\end{array}$ \\
\hline$\overline{\text { Age, mean (SD) }}$ & $73.2(11.7)$ & $67.2(13.1)$ & $78.9(9.1)$ & $74.4(8.6)$ & $73.0(11.4)$ & $73.5(12.3)$ \\
\hline Sex, M:F & $49: 36$ & $25: 7$ & $13: 15$ & $11: 14$ & $36: 21$ & $13: 15$ \\
\hline Patients with follow-ups, $n(\%)$ & $72(84.7 \%)$ & $26(81.3 \%)$ & $26(92.9 \%)$ & $20(80 \%)$ & $52(91.2 \%)$ & $20(71.4 \%)$ \\
\hline
\end{tabular}

Table 2C

Patient Demographics (Cases with Initial and Final Rating)

\begin{tabular}{lccccccc}
\hline & & \multicolumn{3}{c}{ Referral Source } & & \multicolumn{2}{c}{ Target Program } \\
\cline { 3 - 5 } \cline { 6 - 7 } & Total Sample & Acute Care & LTC & Community & & BNU & VBM \\
& $n=70$ & $n=24$ & $n=26$ & $n=20$ & & $n=50$ & $n=20$ \\
\hline Age, mean (SD) & $72.6(11.1)$ & $65.1(11.6)$ & $77.9(8.6)$ & $74.8(8.3)$ & & $72.4(11.0)$ & $73.2(11.5)$ \\
Sex, M:F & $40: 30$ & $19: 5$ & $12: 14$ & $9: 11$ & $31: 19$ & $9: 11$ \\
\hline
\end{tabular}

years old. There was no significant difference in age between patients referred from LTC and the community $\left(\mathrm{p}_{3}=0.2480\right)$. There was also no significant difference in age between patients referred to each of the two target programs, i.e., VBM versus BNU $(p=0.7511)$. 
Table 3

Distribution Across Target Programs by Referral Source

\begin{tabular}{lccc}
\hline Referral Source & \multicolumn{2}{c}{ Target Program $(n, \%)$} & Total, $(n)$ \\
\cline { 2 - 3 } & BNU & VBM & \\
\hline Acute Care & $25(71.4 \%)$ & $10(28.6 \%)$ & 35 \\
Long Term Care & $23(76.7 \%)$ & $7(23.3 \%)$ & 30 \\
Community & $14(46.7 \%)$ & $16(53.3 \%)$ & 30 \\
Total & $62(65.3 \%)$ & $33(34.7 \%)$ & 95 \\
\hline
\end{tabular}

There were significantly more men referred from acute care compared to LTC $\left(\mathrm{p}_{1}=0.0345\right)$ and the community $\left(\mathrm{p}_{2}=0.0186\right)$. The proportion of men was not significantly different between referrals from the community and LTC $\left(\mathrm{p}_{3}=0.9997\right)$. In addition, there was no significant difference in the proportion of men and women referred to the BNU compared to VBM $(p=0.3468)$.

Table 3 shows the proportion of the 95 patients referred to the BNU versus VBM from acute care, LTC, and the community. There was no difference in proportion of patients referred to the BNU or VBM comparing the community and LTC $\left(\mathrm{p}_{1}=0.0582\right)$ or acute care $\left(\mathrm{p}_{2}=0.067\right)$. In addition, no difference was found between referrals from LTC and acute care $\left(p_{3}=0.63\right)$. Although there were no statistically significant differences in proportion of patients referred to the BNU and VBM from the different referral sources, Table 3 suggests that the profile of referrals from acute care and LTC is characterized by more referrals to the BNU than to VBM, whereas referrals from the community are more equally divided between the BNU and VBM.

\section{Participants entered into VBM}

Among the 95 patients referred to the program, 10 did not enter the program because they were discharged following initial virtual assessment. Five had been referred to VBM and five to the BNU. Five had been referred from the community, three from acute care, and two from LTC. Seven of these patients did not require the program. The plan for the other three was to have them followed by their psychiatrist rather than in VBM. Thus, 85 patients were admitted to VBM during the study period. This sample comprised the group in whom we estimated the characteristics associated with time required to alleviate the need for hospitalization. Table 2B shows the characteristics of these patients.

There was a large association between the three referral sources and age (Cohen's $f=0.55$ ), with older participants referred from LTC and younger
Table 4

Enrolled Patient Distribution Across Target Program by Referral Source

\begin{tabular}{lccc}
\hline Referral Source & \multicolumn{2}{c}{ Target Program $(n, \%)$} & Total, $(n)$ \\
\cline { 2 - 3 } & BNU & VBM & \\
\hline Acute Care & $23(71.9 \%)$ & $9(28.1 \%)$ & 32 \\
Long Term Care & $22(78.6 \%)$ & $6(21.4 \%)$ & 28 \\
Community & $12(48.0 \%)$ & $13(52.0 \%)$ & 25 \\
Total & $57(67.1 \%)$ & $28(32.9 \%)$ & 85 \\
\hline
\end{tabular}

participants referred from acute care. The participants from the community were intermediate in age between those from LTC and acute care. There was a negligible association between age and referral target $(f=0.03)$. There was a medium association between referral source and sex (Cramér's $V_{2 d f}=0.23$ ) and a medium association between referral source and referral target (Cramér's $\left.V_{2 d f}=0.19\right)$. There was a small association between referral target and sex $\left(\mathrm{V}_{1 \mathrm{df}}=0.13\right)$. The referrals from acute care were predominantly male, whereas the referrals from LTC and the community were more equally divided. While referrals from the community were fairly evenly split between the BNU and VBM, referrals from acute care and LTC were predominantly to the BNU (Table 4).

To examine the association between initial rating and referral source, as well as referral to the BNU or VBM, an ordinal logistic model was run including these two referral factors. Patients from the community had significantly higher initial ratings than those from acute care $\left(\mathrm{t}(77)=2.98, \mathrm{p}_{1}=0.0115\right)$, i.e., their ratings were less severe. Initial ratings were not significantly different between LTC and community $(\mathrm{t}$ $\left.(77)=2.15, \mathrm{p}_{2}=0.0523\right)$ or acute care $(\mathrm{t}(77)=0.89$, $\mathrm{p}_{3}=0.3758$ ). For referral target, initial ratings were significantly lower, i.e., more severe, for patients referred to the BNU compared to those referred to VBM (t $(77)=3.974, p=0.0002)$. The violin plot in Fig. 2 shows initial ratings by referral source, i.e., acute care, LTC, and community, as well as referral target to the BNU and VBM for the 85 cases. There was a large association between initial rating and referral source $(f=0.53)$ and with referral target $(f=0.80)$.

\section{Duration in program}

Duration of stay in VBM was based on the 85 participants entered into the program. Of these cases, 40 were discharged with no significant NPS. In addition, one patient had no significant NPS but died of COVID-19 before being officially discharged. 


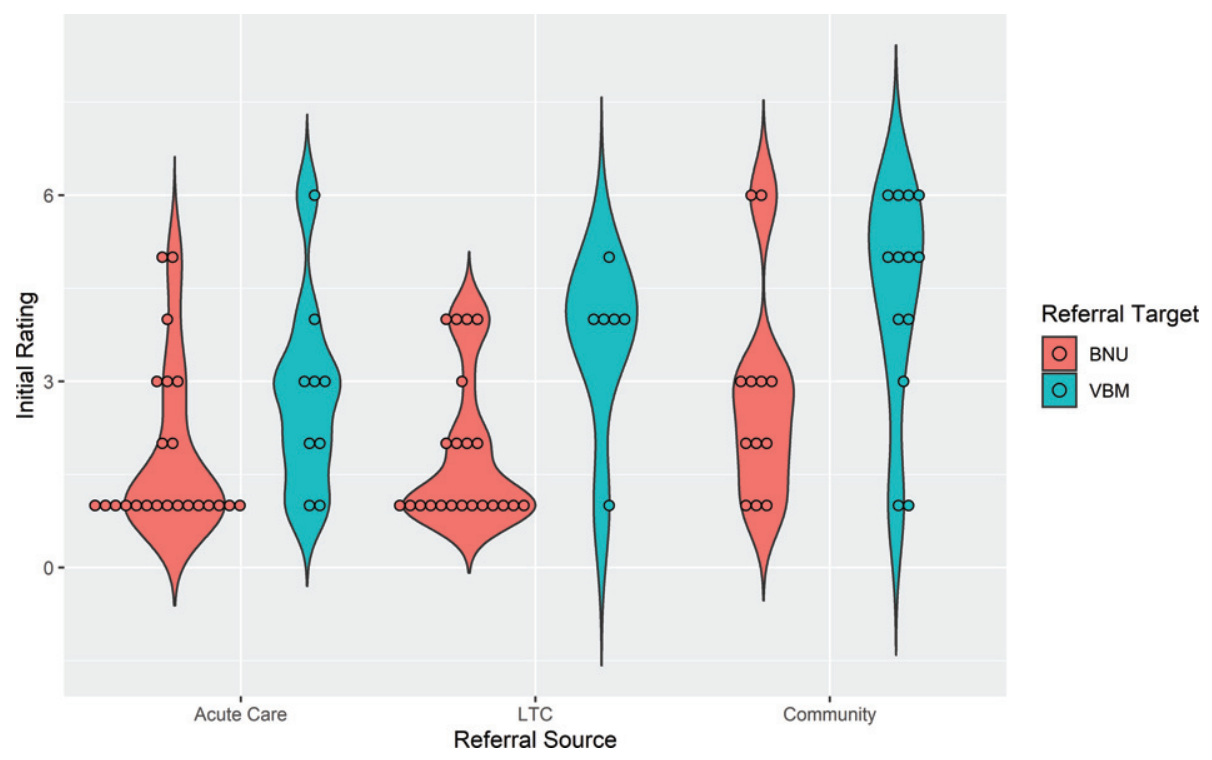

Fig. 2. Violin plot showing initial rating by referral source and referral target for patients admitted to VBM $(n=85)$.

Table 5A

Time to discharge from VBM or BNU waitlist (Rating 7)

\begin{tabular}{lccc}
\hline Duration (days) & $\begin{array}{c}\text { Estimated } \\
\text { Median }\end{array}$ & $\begin{array}{c}\text { Lower } \\
95 \% \text { CL }\end{array}$ & $\begin{array}{c}\text { Upper } \\
\text { 95\% CL }\end{array}$ \\
\hline From Acute Care & 147 & 44 & $\infty$ \\
From LTC & 173 & 104 & 218 \\
From Community & 103 & 67 & $\infty$ \\
To BNU & 173 & 119 & 218 \\
To VBM & 76 & 49 & $\infty$ \\
\hline
\end{tabular}

Another 11 cases were discharged while they still had NPS. These cases were admitted to a specialized neurobehavioral unit $(n=7)$, acute care for management of NPS without further follow-up in VBM $(n=2)$, LTC from acute care without request for follow-up in $\operatorname{VBM}(n=1)$, and acute care for surgery related to a clavicle fracture and acromioclavicular dislocation without subsequent follow-up in VBM $(n=1)$. Table 5A shows median time in days to discharge from VBM or the BNU waitlist (rating 7) by referral source and referral target. Table 5B shows the same data by number of visits. We ran a proportional hazards model to investigate the association between time to discharge (rating 7) with referral source and referral target. Number of follow-up visits and number of days in the program were considered as two measures of time that are relevant to understanding the characteristics of the needs of the patients in the program and the resources required to address the needs.
Table 5B

Number of visits to discharge from VBM or BNU waitlist (Rating 7)

\begin{tabular}{lccc}
\hline Follow-up Visits & $\begin{array}{c}\text { Estimated } \\
\text { Median }\end{array}$ & $\begin{array}{c}\text { Lower } \\
95 \% \text { CL }\end{array}$ & $\begin{array}{c}\text { Upper } \\
95 \% \text { CL }\end{array}$ \\
\hline From Acute Care & 8 & 3 & $\infty$ \\
From LTC & 12 & 4 & $\infty$ \\
From Community & 6 & 3 & $\infty$ \\
To BNU & 12 & 6 & 19 \\
To VBM & 5 & 2 & $\infty$ \\
\hline
\end{tabular}

The proportional hazards model was fit to show the proportion of patients still in the program (rating 1-6) based on the number of visits in the program (Fig. 3, left side). There was a significantly longer duration in the program comparing referrals to $\mathrm{BNU}$ with those to $\operatorname{VBM}(z=2.546, p=0.0109)$. There was no association between number of visits required and referral source $(p>0.52)$. Seventy percent of referrals to VBM were transitioned to discharged at 8 visits. In contrast, $70 \%$ of referrals to BNU were transitioned to discharged at 16 visits.

The proportional hazards model was also fit to show the proportion of patients still in the program (rating 1-6) based on the number of days in the program (Fig. 4, left side). There was a significantly longer duration in the program comparing referrals to $\mathrm{BNU}$ with those to $\operatorname{VBM}(z=2.751, p=0.00595)$. No significant effect of referral source was found $(p>0.87)$ (Fig. 5). 
By referral target

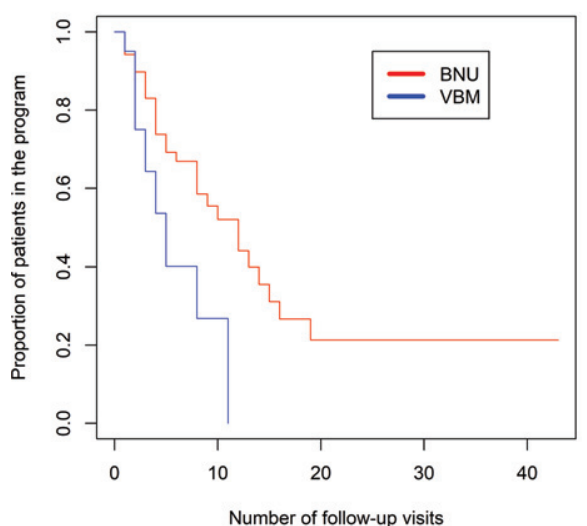

By initial rating

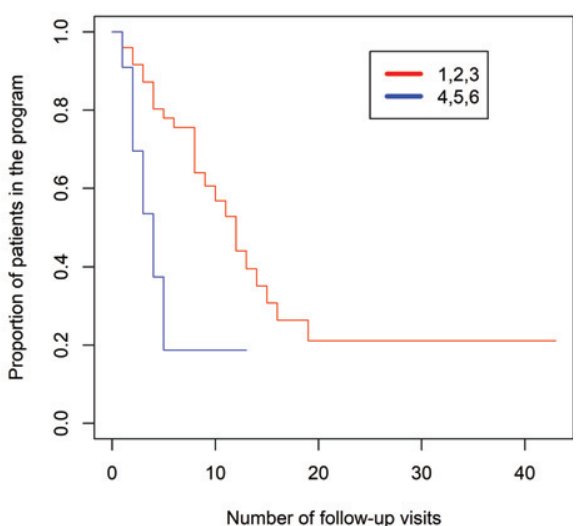

Fig. 3. Kaplan-Meier estimated "survival" plot showing proportion of patients still in the program (rating 1 to 6) based on number of follow-up visits. Plot on left shows patients divided by referral to VBM alone (in blue) or VBM plus BNU (in red). Plot on right shows patients with initial severity rating of 1,2 , or 3 (in red) or 4,5 , or 6 (in blue).

By referral target

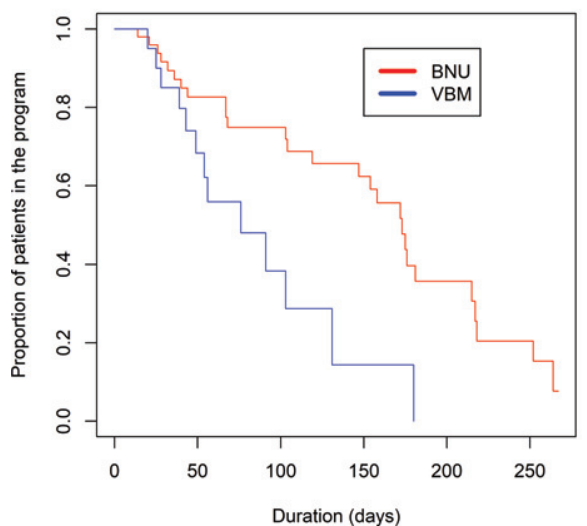

By initial rating

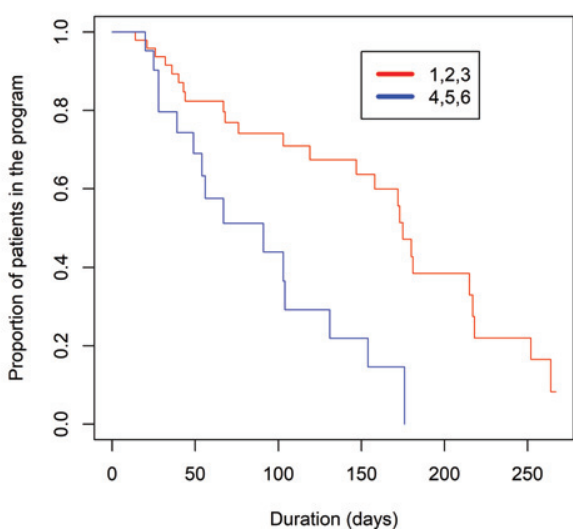

Fig. 4. Kaplan-Meier estimated "survival" plots showing proportion of patients still in the program (rating 1 to 6) based on number of days in the program. Plot on left shows patients divided by referral to VBM alone or VBM plus BNU. Plot on right shows patients with initial severity rating of 1,2 , or 3 (in red) or 4,5 , or 6 (in blue).
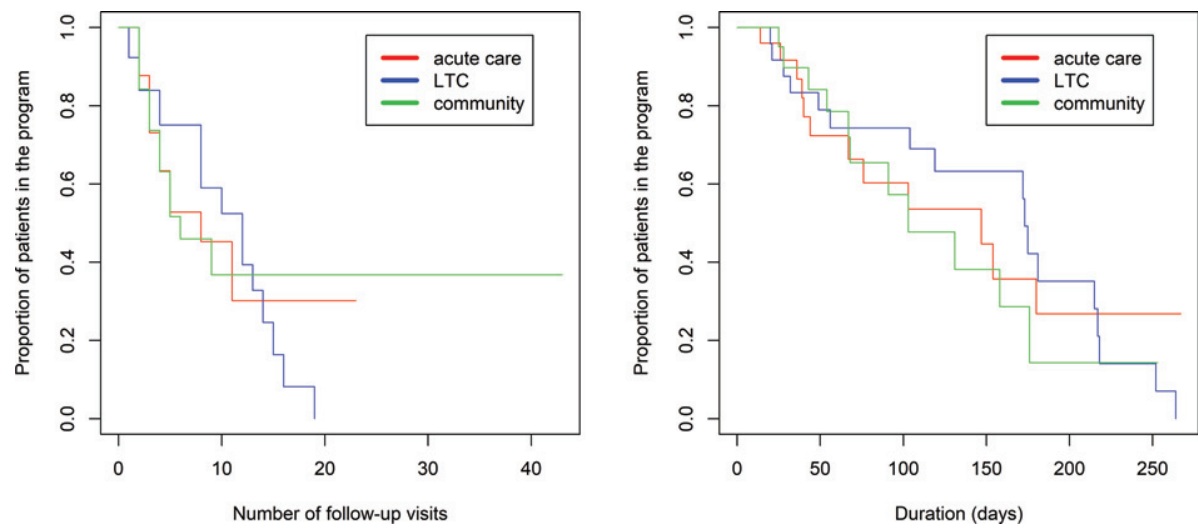

Fig. 5. Kaplan-Meier estimated "survival" plot showing proportion of patients referred from acute care, LTC, and the community who are still in the program (rating 1 to 6) based on number of visits and duration in VBM. 
As shown in the violin plot (Fig. 2), there is negligible overlap of initial rating between participants referred to VBM and those referred to BNU, particularly for participants referred from the community or LTC. Thus, the observed effect might reflect an effect of initial rating. Subsequent models were run with the addition of initial hospitalization priority rating in the model. There was a strong positive association between an initial rating indicating a clear need for hospitalization $(1,2$, or 3$)$ and time to discharge from VBM and no longer needing hospitalization (Rating 7 ), whether time was measured as number of followup visits $(z=3.10, p=0.00193)$ (Fig. 3) or days in the program $(z=2.45, p=0.0143)$ (Fig. 4). With initial rating classification in the model, the effect of referral target was no longer significant for number of follow-up visits $(z=1.026, p=0.30)$ or days in the $\operatorname{program}(z=1.792, p=0.07)$.

\section{Participants with a follow-up rating}

Approximately $75 \%(n=72)$ of the total sample $(n=95)$ had at least one follow-up visit during the study period (Table 2A). Thus, 23 cases did not have a follow-up. Ten were discharged after the initial visit, three were admitted to acute care, two were admitted to a specialized behavioral unit, one died from COVID-19, one was released from prison and admitted to acute care, one was discharged from acute care to the community, and five did not yet have their first follow-up before the end of the study period.

All patients were given an initial rating of 1 to 7 on the Severity Rating Scale. To examine the effect of VBM on reducing need for admission to the BNU, only patients who had both an initial rating and at least one follow-up rating were analyzed. These patients had between 1 and 43 follow-up visits. Although there were 72 cases with a follow-up visit, only 70 had a follow-up rating. The two patients without a followup rating were from acute care and were each seen once after the initial visit. One had delirium and thus the follow-up rating was deferred. For the second case, the staff in acute care who were present at the follow-up visit were unfamiliar with the patient and thus the rating was deferred.

Table 2C shows the characteristics of the patient sample entered into the program and for whom there were both initial and final ratings. There was a large association between the three referral sources and age (Cohen's $f=0.69$ ) with younger participants referred from acute care, older participants referred from LTC, and referrals from the community being intermediate
Table 6

Distribution of Patients with Initial and Final Severity Rating Indicating Need for BNU Admission

\begin{tabular}{lccc}
\hline & \multicolumn{2}{c}{ Target Program } & \\
\cline { 2 - 3 } & $\begin{array}{c}\text { BNU } \\
(n=50)\end{array}$ & $\begin{array}{c}\text { VBM } \\
(n=20)\end{array}$ & $\begin{array}{c}\text { Total, } \\
(n=70)\end{array}$ \\
\hline $\begin{array}{c}\text { Initially requiring BNU } \\
\text { admission }(n, \%)\end{array}$ & $48(96.0 \%)$ & $16(80.0 \%)$ & 64 \\
$\begin{array}{c}\text { Requiring BNU admission } \\
\text { after final rating }(n, \%)\end{array}$ & $19(38.0 \%)$ & $5(25.0 \%)$ & 24 \\
\hline
\end{tabular}

in age between those from LTC and acute care. There was a negligible difference in age due to referral target $(f=0.05)$. There was a medium association between referral source and sex (Cramér's $V_{2 d f}=0.23$ ), a medium association between referral source and referral target $\left(\mathrm{V}_{2 \mathrm{df}}=0.21\right)$, and a small association between referral target and sex $\left(\mathrm{V}_{1 \mathrm{df}}=0.12\right)$. Referrals from acute care were predominantly male whereas referrals from LTC and the community were more equally divided. While referrals from the community were split fairly evenly between the BNU and VBM, referrals from acute care and LTC were predominantly to the BNU. In this sub-sample, there was a large association between initial rating and referral source $(f=0.64)$ and with referral target $(f=0.75)$. Initial rating was lower (greater need for hospitalization) for participants referred from acute care and LTC and higher for participants referred from the community. Participants referred to VBM had higher initial ratings (less need for hospitalization) than those referred to the BNU.

Table 6 shows the distribution of patients referred to the BNU or VBM whose initial severity rating was $1,2,3,4$, or 5 and thus indicated a requirement for admission to the BNU. Ninety-six percent of those referred to the BNU required admission. For those referred to VBM without a parallel referral to the BNU, only $80 \%$ were rated as needing admission to the BNU. Following treatment, $38 \%$ of those referred to the BNU and $25 \%$ of those referred to VBM still required admission.

We compared those who initially needed admission (rating of $1,2,3,4$, or 5 ) to those who needed admission based on the final rating. There was a significant reduction in proportion of patients needing admission to the BNU $(p<0.0001)$. However, there was a significant interaction indicating different effects between patients referred to the BNU and those referred to VBM only $(p=0.0497)$. Further analyses showed that there was a significant change in necessity of admission for patients referred 


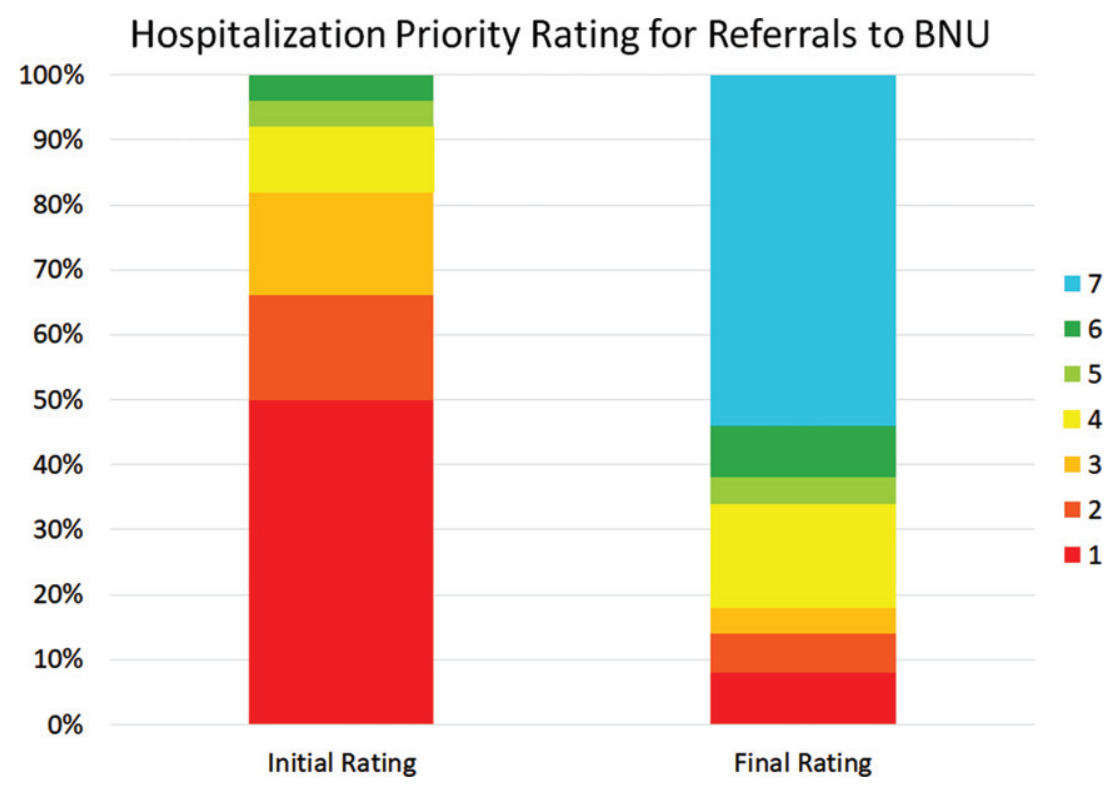

Fig. 6. Initial and latest severity ratings for the cumulative proportion of patients referred to the BNU.

to the $\mathrm{BNU}(p<0.0001)$. However, a slightly smaller but significant absolute difference was observed in patients referred to VBM $(p=0.001)$.

Figure 6 shows the initial and latest severity ratings for the cumulative proportion of patients referred to the BNU. Figure 7 is an alluvial plot showing the initial and latest severity ratings for the cumulative proportion of patients referred to the BNU, as well as movement of patients from the initial to the latest ratings. For these patients, and as indicated above, 96\% $(n=48)$ required admission to the BNU based on an initial severity rating of $1,2,3,4$, or 5 . However, based on the latest rating, only $38 \%(n=19)$ required admission. This represents a reduction in those who required admission to the BNU by $60.42 \%$ (CI: 47-74\%). The proportional reduction in need for admission was calculated as the difference between the number of patients requiring admission at initial rating and the number of patients requiring admission at final rating, divided by the number of patients initially requiring admission.

Figure 8 shows the initial and latest severity ratings for the cumulative proportion of patients referred to VBM. Figure 9 is an alluvial plot showing the initial and latest severity ratings for the cumulative proportion of patients referred to VBM, as well as the movement of patients from the initial to the latest ratings. For these cases, $80 \%(n=16)$ were rated as requiring admission to the BNU based on the initial rating even though no referral to the BNU was made.
Based on the final rating, only $25 \%(n=5)$ required admission. This represents a $68.75 \%$ (CI: 44-89\%) reduction in those who needed admission to the BNU.

The reduction in those who needed admission to the BNU for all cases combined, i.e., regardless of whether they were referred to the BNU plus VBM or only to VBM, was $62.5 \%$ (CI: 51-74\%).

We also examined the reduction in need for admission to the BNU by comparing those who had an initial rating of $1-3$, i.e., those who clearly needed admission, to those who clearly needed admission based on the final rating. For those referred to the $\mathrm{BNU}$, the percentage reduction was $78.05 \%$ [i.e., $\left.100^{*}(41-9) / 41\right]$ and for those refereed to only to VBM, it was $57.14 \%$ [i.e., $100 *(7-3) / 7$ ].

\section{DISCUSSION}

VBM is a novel model of virtual care for assessment and management of severe NPS associated with dementia. In applying this model to patients referred to our specialized inpatient BNU, we successfully reduced the proportion of patients who were in need of admission to this unit by $60.42 \%$. These were the patients with initial severity ratings of 1-5. Prior to the development of VBM, essentially all patients with an application for admission to the inpatient BNU were admitted when a bed became available. Whereas our findings are based 
Hospitalization Priority Rating for Referrals to BNU

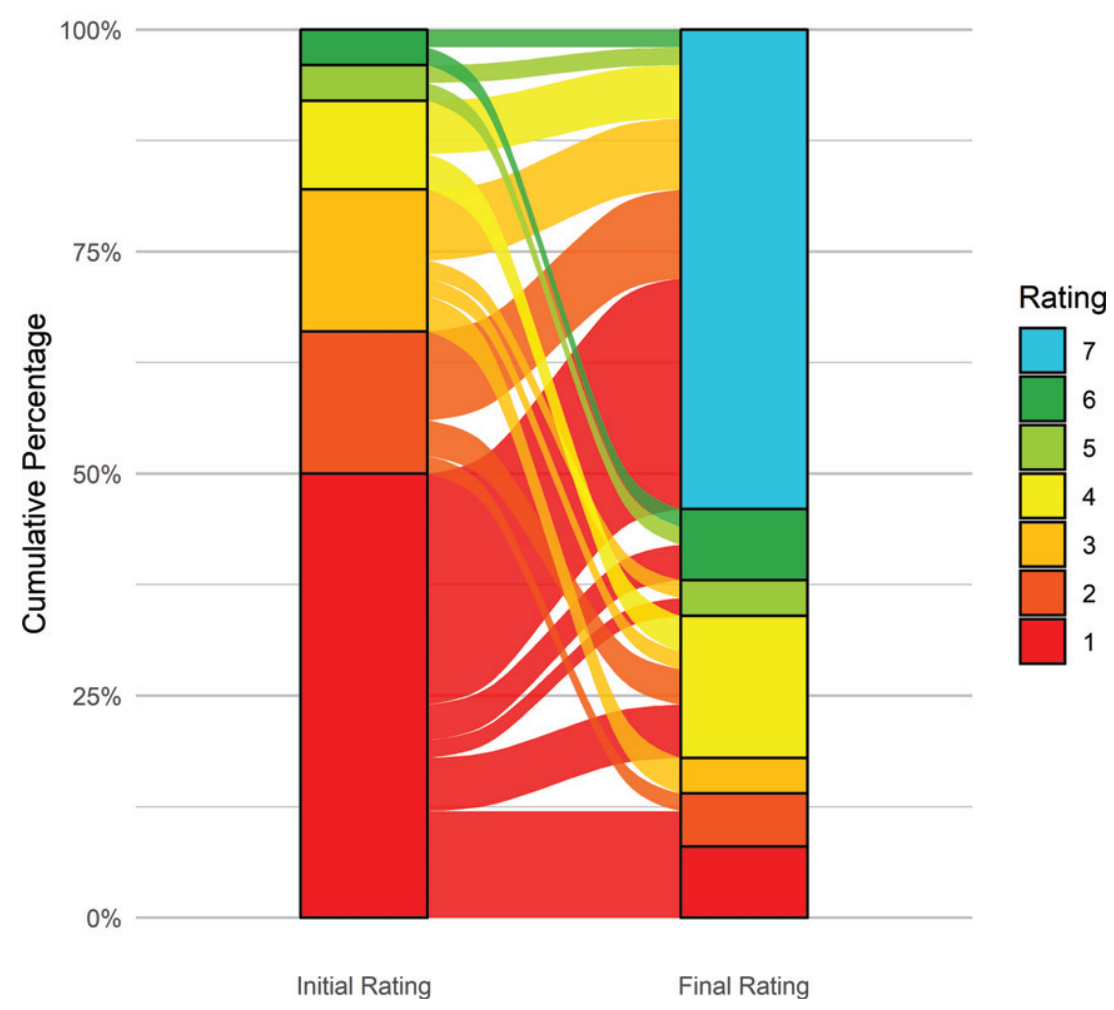

Fig. 7. Alluvial plot showing the initial and latest severity ratings for the cumulative proportion of patients referred to the BNU, as well as the movement of patients from the initial to the latest rating.

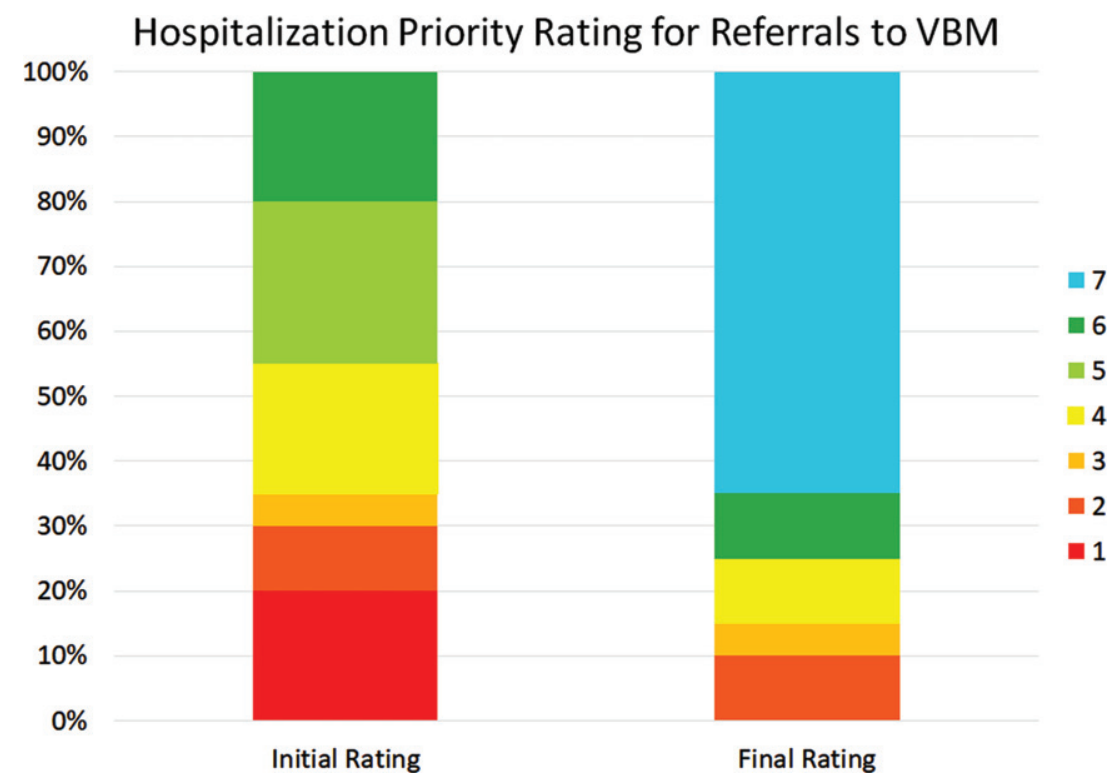

Fig. 8. Initial and latest severity ratings for the cumulative proportion of patients referred to VBM alone. 


\section{Hospitalization Priority Rating for Referrals to VBM}

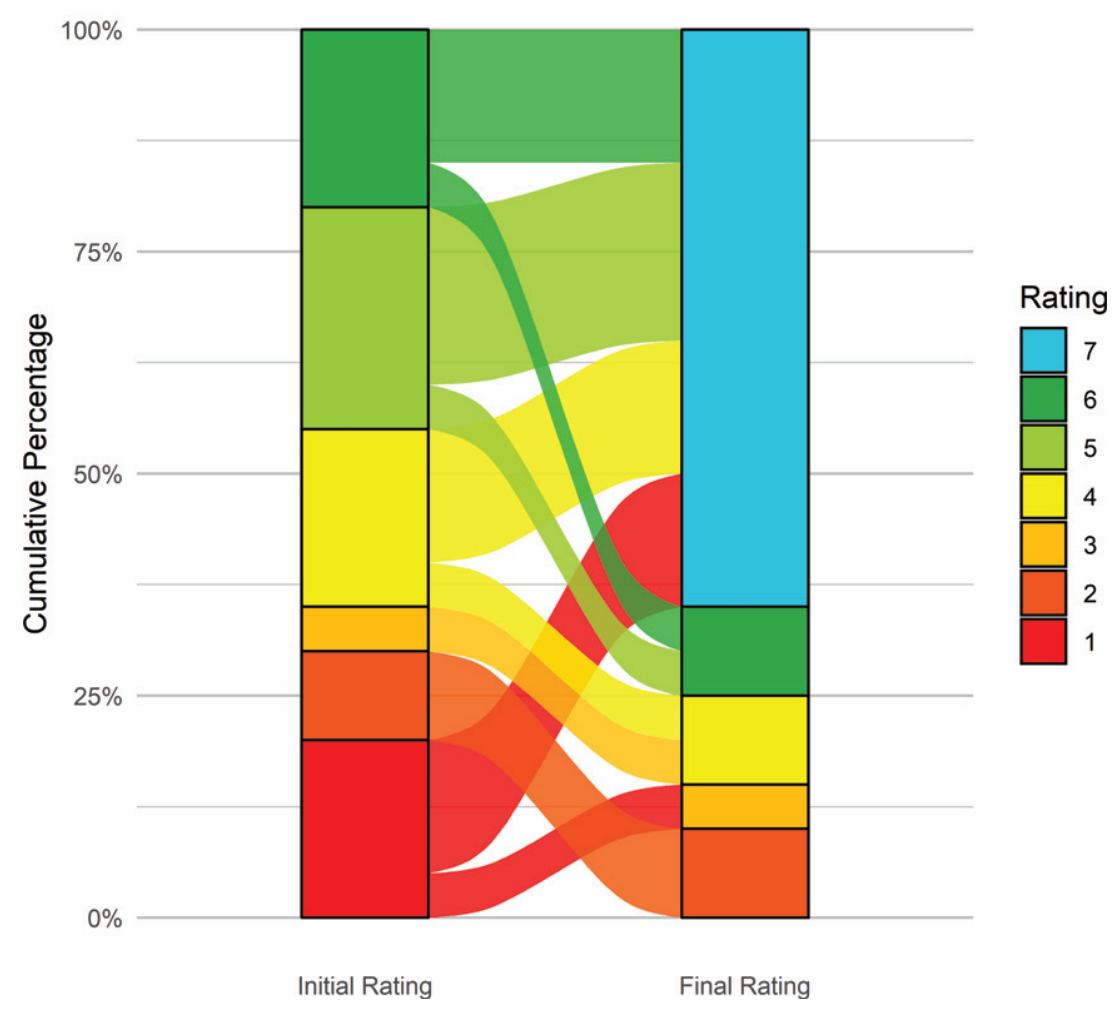

Fig. 9. Alluvial plot showing the initial and latest severity ratings for the cumulative proportion of patients referred to VBM alone, as well as the movement of patients from the initial to the latest rating.

on a single cohort, the $95 \%$ confidence limits for this reduction were $47-74 \%$. This supports the concept that VBM is highly effective for managing the majority of patients demonstrating NPS without a need for physical transfer to a specialized neurobehavioral program. Thus the benefits of VBM include avoiding exposure of patients to unnecessary changes to their environment which could worsen NPS [6], as well as avoiding unnecessary use of limited and costly inpatient hospital resources. Moreover, avoiding transfers facilitates continuity of care. In addition, for community dwelling patients, it is often difficult for caregivers to bring the person they care for to a physician's office when NPS are present. VBM avoids the need for these patients to travel for assessment and management.

In addition to reducing the need for admission of patients referred to the inpatient BNU, we examined the subset of cases referred to VBM without a parallel application to the BNU. Although this was a milder group with less severe NPS compared to the group with an inpatient application, $80 \%$ of cases in this former group had NPS that were severe enough to warrant a need for inpatient admission. This contrasts with $96 \%$ of patients who were rated as needing admission among those referred to the inpatient BNU. In this subgroup with a referral only to VBM, the proportional reduction in patients who needed admission to the inpatient unit, i.e., who had an initial severity rating of $1-5$, was $68.75 \%$ with a $95 \%$ confidence interval of 44-89\%. When considering all referrals to VBM, i.e., those with a parallel referral to the inpatient unit and those without such a referral, the reduction in proportion of patients in need of admission to the inpatient unit was $62.5 \%$ with a $95 \%$ confidence interval of $51-74 \%$.

VBM is in essence a specialized "virtual inpatient unit" that extends beyond the physical walls of the hospital to serve LTC homes, acute care hospitals, and the community. Since this unit is virtual, there are no limitations due to physical space or bed availability. In contrast, the main limitations are staff resources involving physicians, allied healthcare professionals, and administrative support. In addition, access to a 
stable internet connection and computer equipment are limitations in some cases.

Another key feature differentiating VBM from traditional physical inpatient units focused on NPS is the concept of healthcare team. In contrast to a fixed team comprised of healthcare professionals based on a physical inpatient unit at a single institution, teams in VBM are fluid and vary depending on their location. Although there is a core VBM team based at the host site, the extended team includes a partnership between the core VBM group and healthcare professionals in the LTC homes, acute care hospitals, and the community. Since the extended VBM team members work together on individual cases, there is capacity building due to sharing of expertise. This capacity building is further enhanced when teams come together in the assessment and management of additional patients referred from the same LTC homes and acute care hospitals.

Our findings are relevant to program development and planning. First, there was approximately an equal percentage of patients referred to VBM from LTC, acute care, and the community. Thus, VBM is designed to manage patients with NPS across the full spectrum of care. Expansion of VBM, as well as development of comparable programs at other sites, will require attention to the different resources and needs in LTC, acute care, and the community. For example, scheduling patients in LTC and acute care requires development of standard operating procedures to coordinate with several members of a healthcare team and possibly family members or caregivers, whereas scheduling in the community involves coordination primarily with family members or caregivers.

Second, duration of stay in VBM initially appeared to relate to whether patients were referred to VBM alone or to VBM plus the inpatient unit with a longer duration for those with an inpatient referral. However, further analysis suggested that a key predictor of duration in VBM was initial severity of NPS rather than target referral program. Thus, the reason that patients referred to the BNU required a longer treatment period than those referred only to VBM may be that the former group had more severe NPS at the start of therapy. In contrast to the relation between duration in VBM and target referral program, there was no relation between duration of stay and whether patients were referred from acute care, LTC, or the community. Thus, program planning related to duration of stay should focus on initial severity of NPS rather than referral source, i.e., LTC, acute care, or community, or referral target program, i.e., VBM alone versus VBM plus inpatient unit.

Third, there were differences in age and sex based on referral source. There was a large association between age and referral source with younger participants referred from acute care, older participants referred from LTC, and referrals from the community being intermediate in age between those from LTC and acute care. In addition, referrals from acute care were predominantly male whereas those from LTC and the community were more equally divided between males and females. Thus, programs aimed at accepting patients from acute care should be prepared to manage NPS, such as aggression, in a younger population that is predominantly male. Regarding patients referred to the $\mathrm{BNU}$, there were more males than females but this was not significantly different from referrals to VBM alone.

Fourth, consideration should be given to whether there is a difference in referral pattern from acute care, LTC, and the community to VBM versus VBM plus a referral to the inpatient BNU. Although there were no statistically significant differences, the data suggest that referrals from acute care and LTC more often include a referral to both the inpatient BNU and VBM than to VBM alone. In contrast, the data suggest that referrals from the community were more equally divided between these two options. This may reflect a greater tendency for acute care hospitals and LTC facilities to transfer patients out of their facilities as compared to caregivers in the community not wanting an inpatient admission. Although further study is required to confirm this, VBM programs that accept referrals from acute care and LTC should be prepared to address the pressures associated with a higher demand for inpatient admission.

Fifth, approximately $10 \%$ of initial referrals to the VBM program (10 cases) were discharged from the program at the initial visit. This suggests that better screening with triage to other services, as well as education among referral sources about availability of alternate resources, might have redirected these referrals without a formal assessment in VBM.

Although we did not assess costs of the VBM program compared to care on the physical inpatient behavioral unit, it appears that the costs would be considerably less in VBM. Patients are seen in VBM for an initial assessment and follow up appointments. The entire team consists of two staff physicians, behavioral neurology fellows, a nurse, a clinical navigator, and a pharmacist. There is also administrative support. This contrasts with the more extensive 
resources on our inpatient unit which operates 24 hours per day, seven days a week, and includes a team of registered nurses, advanced practice nurse, registered practical nurses, personal support workers, three staff physicians (behavioral neurologist, geriatric psychiatrist, primary care physician), behavioral neurology fellows, allied health care professionals including a neuropsychologist, occupational therapist, physiotherapist, pharmacist, recreation therapist, speech-language pathologist, social worker, dietician, and administrative support. Other considerations are costs of the physical space, overhead support including pharmacy for distribution and costs of medications, as well as back office supports such as finance department, food, and nutrition costs. Patients seen in VBM from long-term care who do not require a transfer to the Behavioural Neurology Unit or other acute care bed remain in one bed. This contrasts with their being admitted to an inpatient unit bed in which case they would be occupying two beds since their long-term-care bed would be held. Thus, a savings of bed days could be calculated. In addition, the costs of patients being sent to acute care hospitals and emergency departments for treatment of neuropsychiatric symptoms of dementia while waiting for a bed on the inpatient unit needs to be factored in as well. Moreover, community dwelling patients in crisis while waiting for a bed on an inpatient unit often trigger 911 calls, which draw on police and ambulance resources. VBM may serve to reduce these crises. However, to address the issue of the cost effectiveness of the VBM program, a direct assessment of costs in VBM compared to a physical inpatient behavioral unit should be formally studied.

Key factors related to the success of the VBM program include the pharmacological and nonpharmacological approaches that are utilized in the program. For a review of general principles and overall management approach that we take in VBM, see Wolf, Goldberg, and Freedman [4]. A few key points will be mentioned here to illustrate general principles of our approach.

\section{Pharmacological approach}

A major point is that the evidence for choosing which medications to prescribe for NPS in dementia is limited. It is therefore often necessary to improvise in the pharmacological management. A general approach that we have used with good results is to borrow pharmacological strategies from psychiatric conditions that may manifest in the absence of dementia, such as mood disorders and obsessive compulsive disorder, when similarities exist between these disorders and the target neuropsychiatric symptoms in dementia. For example, patients with repetitive, disruptive, compulsive-like behaviors may improve with medications used for obsessive compulsive disorder. Moreover, when newer drugs fail, it is worth considering older medications that are used less commonly. For example, we had success in using clomipramine in patients with compulsive-like behaviors within the context of dementia who did not respond to selective serotonin reuptake inhibitors [22].

Another general principle is to select medications for NPS based on their side effect profile. For example, medications for agitation and aggression [23] that have sedation as a potential side effect, such as gabapentin, may be helpful in patients with NPS who also have difficulty sleeping. Thus for gabapentin, we divide the dose and often administer the last dose at bedtime when sleep is poor. For patients with NPS and poor appetite, we may consider atypical antipsychotics, especially olanzapine, since these medications may improve appetite. Mirtazapine, an antidepressant, may also increase appetite.

Other general principles relate to awareness of drug interactions in which one drug for NPS may increase or decrease the levels of other drugs given concomitantly, as well as that medication for NPS should be started in low doses and that the maximum dose of medication used in patients with dementia is often lower than in younger patients. In addition, patients often present with multiple NPS, such as physical aggression, hallucinations, and depression. In these cases, it is important to prioritize which behavior to target first since treatment may differ depending on the target behavior. Finally, patients may need combinations of medication to effectively treat NPS in dementia. The aim should be to use the lowest dose possible, although relatively high doses are required in some cases.

\section{Nonpharmacological interventions}

Clinicians working from a nonpharmacological approach utilize evidence-based tools to assess NPS in persons living with dementia and to identify possible triggers for these symptoms. These could include unmet needs such as hunger, pain, unpleasant stimuli in the environment such as loud noises, psychological factors such as loneliness, or lack of purpose, and ineffective approaches from the care 
team. Factors guiding choice of behavioral interventions include the patient's background, motivation, strengths, weaknesses, and physical limitations that might affect the interventions [4].

Building on the information collected, Behaviour Supports Clinicians develop a patient centered intervention and care plan and provide coaching and guidance throughout implementation. Examples of nonpharmacological interventions used in VBM include the Gentle Persuasive Approach [24], validation therapy [25], redirecting patients, modifications to eliminate environmental triggers, and Montessoribased activities [26]. In addition, tasks may be adapted to meet a person's capabilities.

Studies have shown that non-pharmacological interventions may provide positive results in the management of NPS [27]. In the majority of patients referred to the Behaviour Supports Services, nonpharmacological interventions are often the first line of intervention. However for some patients, there is a need for pharmacological intervention to complement nonpharmacological interventions. Thus, the focus of VBM has been on combining pharmacological and behavioral interventions. This requires collaboration among the health care teams in LTC, acute care, and the community, as well as the patient's family and caregivers.

\section{Limitations}

There are limitations that should be noted. First, the study sampled one cohort over a specified period. Replication at our site is needed to demonstrate consistent efficacy. Second, replication is needed in other centers to demonstrate that the VBM model is scalable and generalizable to other centers, as opposed to being dependent on factors that may be specific to our institution. Third, the Severity Rating Scale was scored by the treating physicians involved in the clinical assessment and management of the patients. Moreover, they were not blind to the referral source or to other factors that may have influenced the ratings. Although one can argue that the treating physicians are in the best position to rate the patients, future studies should include severity ratings by an independent assessor. In addition, although the rating scale has clear face validity, it should be studied for reliability and validity. Fifth, the sample size was relatively small. Sixth, our study used a pre-post design to describe change in need for admission and did not include a control group that would have allowed characterization of change due to other factors such as the natural course of patients presenting with NPS or utilization of alternate health care utilization.

\section{Conclusion}

VBM represents an innovative model of virtual care designed to assess and manage patients with NPS while they remain in their own familiar environment. VBM is a virtual neurobehavioral unit without walls that serves the needs of patients in LTC, acute care, and the community. Among patients referred to a specialized inpatient neurobehavioral unit, VBM successfully reduced the proportion who needed admission by $60.42 \%$. VBM is a game changer that can reduce preventable emergency department visits and acute care hospital admissions, which are existing pressures further exacerbated by COVID-19. In addition, VBM is a scalable model of novel virtual care that can be adopted worldwide.

\section{ACKNOWLEDGMENTS}

We gratefully acknowledge Rohila Hashimi for her assistance with data collection, Jordanne Holland for assistance with implementation of VBM, and Robin Mitchell and Robin Krishnan for assistance in obtaining data related to the inpatient Behavioural Neurology Unit. VBM was supported in part by the Toronto Central Local Health Integrated Network. M. Freedman receives support from the Saul A. Silverman Family Foundation as a Canada International Scientific Exchange Program and Morris Kerzner Memorial Fund. E. Teleg is the D H Gales Family Foundation Fellow in Behavioural Neurology and was supported by the DH Gales Family Foundation. In-kind support was provided from the Baycrest Sam \& Ida Ross Memory Clinic in the form of nursing, managerial, administrative and IT resources.

Authors' disclosures available online (https:// www.j-alz.com/manuscript-disclosures/21-5403r1).

\section{REFERENCES}

[1] World Health Organization [https://www.who.int/newsroom/fact-sheets/detail/dementia]

[2] Lyketsos CG, Carrillo MC, Ryan JM, Khachaturian AS, Trzepacz P, Amatniek J, Cedarbaum J, Brashear R, Miller DS (2011) Neuropsychiatric symptoms in Alzheimer's disease. Alzheimers Dement 7, 532-539.

[3] Cerejeira J, Lagarto L, Mukaetova-Ladinska EB (2012) Behavioral and psychological symptoms of dementia. Front Neurol 3, 73.

[4] Wolf MU, Goldberg Y, Freedman M (2018) Aggression and agitation in dementia. Continuиm 24, 783-803. 
[5] Cohen-Mansfield J, Ray CA, Hai T, Marcu C, Callahan BL, Freedman M (2017) The ultimate outlier: Transitional care for persons with dementia and BPSD. Curr Alzheimer Res 14, 969-977.

[6] Ryman FVM, Erisman JC, Darvey LM, Osborne J, Swartsenburg E, Syurina EV (2019) Health effects of the relocation of patients with dementia: A scoping review to inform medical and policy decision-making. Gerontologist 59, e674-e682.

[7] Leff B, Burton L, Mader SL, Naughton B, Burl J, Inouye SK, Greenough WB, 3rd, Guido S, Langston C, Frick KD, Steinwachs D, Burton JR (2005) Hospital at home: Feasibility and outcomes of a program to provide hospital-level care at home for acutely ill older patients. Ann Intern Med 143, 798-808.

[8] Lee JH, Kim JH, Jhoo JH, Lee KU, Kim KW, Lee DY, Woo JI (2000) A telemedicine system as a care modality for dementia patients in Korea. Alzheimer Dis Assoc Disord 14, 94-101.

[9] Ryan PP, Hawkins KL, Altman S, Granatowski L, Shy BD, Long J, Hanratty R (2021) A novel virtual hospital at home model during the coronavirus disease (COVID-19) pandemic. Infect Control Hosp Epidemiol 42, 1140-1142.

[10] Francis NA, Stuart B, Knight M, Vancheeswaran R, Oliver C, Willcox M, Barlow A, Moore M (2021) Predictors of clinical deterioration in patients with suspected COVID-19 managed in a 'virtual hospital' setting: A cohort study. BMJ Open 11, e045356.

[11] Sitammagari K, Murphy S, Kowalkowski M, Chou S-H, Sullivan M, Taylor S, Kearns J, Batchelor T, Rivet C, Hole C, Hinson T, McCreary P, Brown R, Dunn T, Neuwirth Z, McWilliams A (2021) Insights from rapid deployment of a "virtual hospital" as standard care during the COVID-19 pandemic. Ann Intern Med 174, 192-199.

[12] Leff B, Burton L, Bynum JW, Harper M, Greenough WB, Steinwachs D, Burton JR (1997) Prospective evaluation of clinical criteria to select older persons with acute medical illness for care in a hypothetical home hospital. JAm Geriatr Soc 45, 1066-1073.

[13] Leff B, Burton L, Guido S, Greenough WB, Steinwachs D, Burton JR (1999) Home hospital program: A pilot study. $J$ Am Geriatr Soc 47, 697-702.

[14] Leff B, Montalto M (2004) Home hospital-toward a tighter definition. J Am Geriatr Soc 52, 2141.

[15] Mader SL, Medcraft MC, Joseph C, Jenkins KL, Benton N, Chapman K, Donius MA, Baird C, Harper R, Ansari Y, Jackson JA, Schutzer W (2008) Program at home: A Veterans Affairs Healthcare Program to deliver hospital care in the home. J Am Geriatr Soc 56, 2317-2322.
[16] Leff B (2009) Defining and disseminating the hospital-athome model. CMAJ 180, 156-157.

[17] Cryer L, Shannon SB, Van Amsterdam M, Leff B (2012) Costs for 'hospital at home' patients were 19 percent lower, with equal or better outcomes compared to similar inpatients. Health Aff (Millwood) 31, 1237-1243.

[18] Federman AD, Soones T, DeCherrie LV, Leff B, Siu AL (2018) Association of a bundled hospital-at-home and 30-day postacute transitional care program with clinical outcomes and patient experiences. JAMA Intern Med 178, 1033-1040.

[19] Shepperd S, Iliffe S, Doll HA, Clarke MJ, Kalra L, Wilson AD, Goncalves-Bradley DC (2016) Admission avoidance hospital at home. Cochrane Database Syst Rev 9, CD007491.

[20] Levine DM, Ouchi K, Blanchfield B, Saenz A, Burke K, Paz M, Diamond K, Pu CT, Schnipper JL (2020) Hospital-level care at home for acutely ill adults: A randomized controlled trial. Ann Intern Med 172, 77-85.

[21] Ricauda NA, Bo M, Molaschi M, Massaia M, Salerno D, Amati D, Tibaldi V, Fabris F (2004) Home hospitalization service for acute uncomplicated first ischemic stroke in elderly patients: A randomized trial. J Am Geriatr Soc 52, 278-283.

[22] Furlan JC, Henri-Bhargava A, Freedman M (2014) Clomipramine in the treatment of compulsive behavior in frontotemporal dementia: A case series. Alzheimer Dis Assoc Disord 28, 95-98.

[23] Davies SJ, Burhan AM, Kim D, Gerretsen P, Graff-Guerrero A, Woo VL, Kumar S, Colman S, Pollock BG, Mulsant BH, Rajji TK (2018) Sequential drug treatment algorithm for agitation and aggression in Alzheimer's and mixed dementia. J Psychopharmacol 32, 509-523.

[24] Hung L, Son C, Hung R (2019) The experience of hospital staff in applying the Gentle Persuasive Approaches to dementia care. J Psychiatr Ment Health Nurs 26, 19-28.

[25] Feil N (1993) The validation breakthrough: Simple techniques for communicating with people with "Alzheimer'stype dementia”, Health Professions Press, Baltimore, MD.

[26] Sheppard CL, McArthur C, Hitzig SL (2016) A systematic review of Montessori-based activities for persons with dementia. J Am Med Dir Assoc 17, 117-122.

[27] de Oliveira AM, Radanovic M, de Mello PCH, Buchain PC, Vizzotto ADB, Celestino DL, Stella F, Piersol CV, Forlenza OV (2015) Nonpharmacological interventions to reduce behavioral and psychological symptoms of dementia: A systematic review. Biomed Res Int 2015, 218980. 